\title{
MHD Models of Planetary Nebulae: Review
}

\author{
Guillermo García-Segura \\ Instituto de Astronomía-UNAM, Apdo Postal 877, Ensenada, 22800 \\ Baja California, Mexico
}

\section{Introduction}

When we discuss about MHD effects in planetary nebulae (PNe), there naturally arises a basic question: which magnetic field do we study? One possibility is the ISM magnetic field (e.g. Heiligman 1980), even more if we are concerned with moving PNe (e.g. Soker \& Dgani 1997). The next possibility is the internal or stellar magnetic field (Gurzadian 1962). It is important to start this review by quoting Aller (1958): "It has been pointed out by Minkowski and others that the structural appearance of many planetary nebulae strongly suggest the presence of magnetic fields. It seems unlikely that such magnetic fields are produced ab initio in the nebular shell. Rather, they must have existed in the outer envelope of the parent star. Certain red giants stars with magnetic fields may evolve in such a way that the expansion of the shell is largely governed by the presence of such a field. Magnetic effects may actually be more important than gas pressure differentials and radiation pressure in controlling the evolution of a planetary nebula".

The structural appearance of PNe display a rich variety of shapes, and have been cataloged in a series of morphological classes: bipolar, elliptical, pointsymmetric, irregular, spherical and quadrupolar (Chu, Jacoby \& Arendt 1987; Schwarz, Corradi \& Melnick 1992; Manchado et al. 1996ab). In contrast, except for a few cases, dust shells around AGB stars do not show signs of asphericity (Bujarrabal \& Alcolea 1991; Kahane \& Jura 1994). Thus, during the transition from the AGB to the post-AGB phase, one or more physical processes responsible for the shape of these objects must be initiated. The origin of aspherical nebulae still remains as one of the fundamental problems of PNe formation and evolution (see reviews by Pottasch 1984 and by Iben 1993).

However, supernova remnants (SNRs), which usually present morphologies extremely similar to PNe (e.g., Gaensler 1998, Fig. 1, 7, 11), have been commonly related with magnetic shaping. Obviously, this is due to the easy detection of magnetic fields in SNRs. The SNR field has evolved largely during past decades on the light of the MHD. Pionering work by Rees \& Gunn (1974), followed up by more sophisticated models (e.g. Kennel \& Coroniti 1984; Begelman \& Li 1992) arrived to the concept of a pulsar wind bubble, in which, a relativistic pulsar wind containing a toroidal magnetic field (the fast wind) forms a surrounding bubble where the relativistic particles and the magnetic flux are accumulated (the hot bubble). Both regions are separated by a strong standing MHD shock (the reverse shock). Around the bubble, the material previously ejected by the explosion (the slow wind) is compressed in a thin shell (the swept-up shell). Both regions are separated by a contact discontinuity. The magnetic field inside 
the pulsar wind bubble is expected to be predominantly toroidal as a result of winding by the pulsar's rotation. The reader can clearly see the analogy with the two-wind model proposed by Kwok, Purton \& Fitzgerlad (1978) for PNe. In this sense, the matter ejected in the explosion will be identified as the superwind of PNe. Are PNe non-relativistic versions of SNRs?

Recently, particular emphasis is being given to the explanation of aspherical planetary nebulae (PNe) due to the effects of the stellar magnetic field (e.g. see reviews by Livio 1997; and by López 1997, these proceedings). In this approach, Chevalier \& Luo (1994) have explored the effects of a rotating star with a magnetized, fast wind on the formation of aspherical bubbles, following a two-wind model scenario. They obtained aspherical steady-state PNe structures. More recently, with 2-D MHD simulations, Różyczka \& Franco (1996) found that the time-dependent evolution of a magnetized shocked wind-region has a very complex behavior, and collimated outflows with jet-like features can be created. The 3-D computations performed by García-Segura (1997) corroborates that the magnetized shocked gas indeed creates jets, and the only difference between the 2-D and 3-D results is that the collimated outflow in 3-D is, as expected, likely subject to kink instabilities. Thus, magnetic effects could be responsible for the generation of aspherical PNe as well as collimated flows (jets) observed in some nebulae.

In this paper, we focus on some features that are addressed by several works on MHD. In $\S 2$ we review different scenarios for the origin of magnetized winds. $\S 3$ discusses the production of axisymmetric flows. $\S 4$ describes the confinement of flows and the production of jets and ansae. $\S 5$ address the linearly encreasing kinematics of the collimated outflows, $\S 6$ give solutions for the special case of point-symmetric nebulae and $\S 7$ address solutions for the periodic shells around $\mathrm{PNe}$ and proto-PNe.

\section{The magnetized wind origin}

Four major scenarios with three different types of wind driving mechanisms can be clearly identify:

\section{A. Radiation driven wind + B-field : single or wide binary}

This solution incorporates a magnetic field in the classical theory of Castor, Abbott \& Klein (1975). It works for single stars, however, it is also appropriate for wide binary systems. Analytical solutions can be found in Ignace, Cassinelli \& Bjorkman (1998), for the limit of weak magnetic fields, in which the fields are unimportant in accelerating the flow. Works under this scenario include Chevalier and Luo (1994), Różyczka \& Franco (1996), García-Segura (1997), García-Segura et al. (1999, 2001), García-Segura \& López (2000), Gardiner \& Frank (2001), Dwarkadas et al. (2002). Solutions including a dipole magnetic field can be found in Matt et al. (2000).

B. Magnetic Field driven wind: single or wide binary

In this scenario, the wind is driven by magnetic pressure, and is the B-field the responsible for the mass-loss rate. The main idea of this scenario is that the field is generated at the stellar interior, which emerges through 
magnetic buoyancy to the surface. This solution also works for wide binary systems. Works under this scenario include for example Pascoli (1992) and Blackman et al. (2001a).

\section{Classical binary model: accretion in the secondary}

The theory of MHD launched winds from accretion disk, where toroidal magnetic fields become finally dominant (e.g. Contopoulos 1995) is applied in this scenario. Several solutions differs in the accretion mode: accretion of the primary wind onto the secondary (e.g. Morris 1987, Mastrodemos \& Morris 1998); Roche lobe overflow (e.g. Livio, Salzman, \& Shaviv 1979; Livio \& Soker 1988). See also review by Soker in these proceedings.

D. Alternative binary model: accretion in the primary after common envelope evolution

As in scenario C, magnetized winds are espected to be launched from an accretion disk. In this case, the disk is formed in the primary star. Recent works on this scenario are Reyes-Ruiz \& López (1998) and Blackman et al. (2001b).

In either cases (scenarios A to D) the result is a magnetized wind, in which the toroidal component is dominant. The degree of self-collimation and the appearance of a jet, depends on several parameters.

\section{Axisymmetric flows caused by a fast, magnetized wind}

Axisymmetric flows can be produced by a magnetized wind with or without the existence of an equatorial density enhancement (EDE) (Begelman \& Li 1992, Chevalier \& Luo 1994, Różyczka \& Franco 1996, García-Segura 1997, GarcíaSegura et al. 1999, Matt et al. 2000). Solutions for the formation of EDEs by stellar rotation can be found in Bjorkman and Cassinelli (1993), Ignace et al. (1996, 1998), and by dipole magnetic fields in Matt et al. (2000).

Since the magnetic field at the surface of a post-AGB star is transported out by its wind, and because of stellar rotation, the magnetic field in the wind is dominated by a toroidal component (e.g., Fig. 1 in Ignace et al. 1998). The toroidal field carried by the fast wind can constrain the motion of the flow and an elliptical or bipolar nebula is produced even if the slow wind is spherically symmetric. The resulting toroidal field has a magnetic tension associated with it (Chevalier \& Luo 1994; Contopoulos 1995). Thus, the general effect of the magnetic tension is the elongation of the nebula in the polar direction. The mechanism responsible for the elongation was described by Różyczka \& Franco (1996), and only the basic points are listed here. First, the outer part of the magnetized shocked wind region becomes magnetically rather than thermally supported, i.e., the magnetic energy density becomes larger than the thermal energy density. The latter decreases due to the expansion of the nebula and due to the fact that work must be done against the tension of the toroidal field. Second, the tension of the toroidal field slows down the expansion in the direction perpendicular to the symmetry axis, while the expansion in the direction parallel to the axis proceeds almost unimpeded. Third, a flow in the shocked wind region 
toward the symmetry axis is initiated, leading to the formation of stagnation regions at the rotational axis. Note that at the reverse shock, there is a large gradient in the magnetic pressure because the toroidal field at the rotation axis is null in the free expanding wind.

\section{Confinement of flows: jets and ansae by pinch effect}

The interaction of the two winds generates a wind-shocked region where the tension of the compressed B-field generates an important new feature: a pair of flows are induced (one at each hemisphere), that move the gas toward the polar regions and create a pair of collimated outflows at the poles (Różyczka \& Franco 1996). As a consequence and because of the hoop stress of the toroidal field, a flow in the shocked wind region toward the symmetry axis is initiated and maintained, leading to the formation of stagnation regions at the axis and to the formation of jets. Note that this mechanism works in a similar fashion to that of magnetic confinement experiments in plasma laboratories (e.g., Tokamaks, plasma guns). The pinch effect was introduced for first time by Pascoli (1992) in the context of $\mathrm{PNe}$.

The gas that arrives at the polar regions of the nebula can form relatively dense regions, depending of the radiative cooling conditions (García-Segura \& López 2000), which may be identified with jets. Although the hoop stress is always present in this type of flows, not always the conditions are in favor to form observable jets. Such is the case of fast winds with very low mass-loss rates $\left(10^{-8}-10^{-9} \mathrm{M}_{\odot} \mathrm{yr}^{-1}\right)$. In such conditions, the fast wind piles up at the poles, but the radiative cooling, proportional to $n^{2}$, is not sufficiently strong to allow the formation of visible jets, and ansae-like structures are formed instead. The result of varying the mass loss rate of the fast wind from large values to lower values is quite important for jet formation/detection (García-Segura \& López 2000).

Magnetically confined plasmas by toroidal fields can develop kink, sausage or neck instabilities (e.g., Jackson 1962), which can easily lead to the formation of blobs along the collimated plasma flow, giving the appearance of episodic jets. Another possibility is the MHD Kelvin-Helmholtz instability (Chandrasekar 1961) which, given the conditions of the collimated flow, is very likely to appear as well, probably combined with kink and neck instabilities.

\section{Linearly increasing kinematics of the collimated outflows}

A comparison of the morphologies in observed nebulae with those resulting from the computed models provides a first test of the validity of the model. A second verification can be performed using the kinematics of nebulae and, specifically, with the fast outflows/jets that have been reported for several PNe during this last decade. Examples with fast outflows $\left(\sim 500 \mathrm{~km} \mathrm{~s}^{-1}\right.$ or higher $)$ are He 3-1475 (Riera et al.1995) and MyCn 18 (Bryce et al.1997). The observed large velocity can hardly be explained by invoking hydrodynamical effects alone (Frank et al. 1996), since they are above the critical velocity of $\sim 150 \mathrm{~km} \mathrm{~s}^{-1}$ for which interstellar shocks become adiabatic. Magnetohydrodynamical effects, on the other 
hand, are very efficient in forming these fast collimated outflows, as discussed in the previous sections.

A comparison of a MHD computation with the case of MyCn 18 (Bryce et al. 1997) is shown in García-Segura et al. (1999). The computed model agrees quite well with figure 3a in Bryce et al. (1997), although the model was not specifically intended to reproduce this nebula. The most remarkable feature is the approximately linear increase of the expansion velocity along the jet, that matches the actual echelle observations. This accelerative behavior is produced by the relaxation of the magnetic pressure in the shocked region.

\section{Solutions for point-symmetry}

A particularly intriguing case in PNs morphologies are those that display pointsymmetric structures. At first view, the point-symmetric morphological class does not look very important. But, a careful inspection to the statistically, complete sample of the IAC morphological catalog (Manchado et al. 1996a) reveals 40 objects with some degree of point-symmetric features, which represent $19 \%$ of the total list (215) with a well defined morphology. Note that many of these nebulae are not classified as point-symmetric. In fact, they have been well classified inside the categories of bipolars and ellipticals, such as the case of the Dumbell nebula. This fact points in the direction that point-symmetry is a common feature related to any morphological class, instead of a separate group (Manchado et al. 2000; see also Guerrero, Vázquez \& López 1998). Such a large fraction of the sample $(19 \%)$ suggest that the reason which produces point-symmetry should be very common indeed.

The most convincing solutions up to now, for the formation of pointsymmetric nebulae require the existence of a binary system, and a magnetohydrodynamical collimation of the wind, either for accretion disk winds (Livio \& Pringle 1996; see also Mastrodemos \& Morris 1998, Reyes-Ruiz \& López 1998 and Blackman et al.2001b) or for stellar winds (García-Segura 1997, GarcíaSegura \& López 2000).

Since the toroidal magnetic field carried out by the wind, either stellar or coming from a disk (for the last one see Contopoulos 1995), is always perpendicular to the rotation axis of the central star/disk (see Fig. 3 in García-Segura et al. 2000), any kind of misalignment from the axis (wobbling instability, precession, steady tilt respect to the equatorial density enhancement) will "naturally" produce a point-symmetric nebula. In either case, it is easy to imagine the topology of the magnetic field lines in such scenarios, i.e., multiple rings centered and aligned along the "time-dependent" spin axis of the star/disk.

Close binaries are a necessary condition in Livio \& Pringle (1996), while wide binaries are a sufficient one in García-Segura (1997) and García-Segura \& López (2000). As described in García-Segura et al. (2002), close binaries, either attached or detached, will be in favor of forming bipolars with point-symmetric features, while wide binaries will be in favor of ellipticals with point-symmetric features. 


\section{Periodic Shells}

The multiple concentric rings, or arcs, that were recently discovered by the Hubble Space Telescope around a handful of PNe is one of the most puzzling and unexpected results delivered by the HST (see Kwok, Su, \& Hrivnak 1998; Hrivnak, Kwok \& Su 2001; Terzian \& Hajian 2000 and references therein). The best documented case of these systems of faint concentric rings (hereafter called HST rings) is displayed by NGC 6543 (Balick, Wilson \& Hajian 2000). In reality, they are regularly spaced concentric shells, indicating quasi-periodic events with time intervals, assuming typical expansion velocities of AGB winds, in the range of 500 to 1500 years. Soker (2000) made a critical review of the mechanisms that have been proposed to explain them, and he indicates that mass-loss variations associated with solar-like magnetic cycles are perhaps the best alternative for their origin.

In a more recent paper, Simis, Icke \& Dominik (2001) discuss a new nonmagnetic alternative, and present detailed 1D hydrodynamical simulations for the acceleration of a dusty AGB wind. The stability of this process, however, is difficult to explore at the moment in $2-\mathrm{D}$ or $3-\mathrm{D}$, and it is unclear if the compressions can truly create shells or if they only lead to inhomogeneities in these radiatively driven outflows. Thus, at present, one can consider that dusty flow oscillations (if they really exist) or solar-like magnetic cycles are two possible candidates to generate the HST rings.

In Soker's (2000) view, the magnetic field plays no direct role in the evolution of the AGB wind, however temporal variation in the number of magnetic spots would be able to modify the mass-loss rate. The number of cool spots over the AGB surface, which could be preferred sites for dust formation, is controlled by the magnetic cycle. Thus, given that the mass-loss is driven by radiation pressure on dust grains, the same cycle may also regulate periodic variations in the mass-loss rate. In this interpretation, the magnetic field is a passive player with no dynamical effects, and the mass-loss rate simply follows the spot cycle activity. In addition, to make a logical association with bipolar $\mathrm{PNe}$, he suggests that a stronger magnetic activity could be expected from dynamo amplification in binary systems.

García-Segura et al. (2001) developed a different point of view and explored some of the actual dynamical effects of a solar-like magnetic cycle. The possibility of a solar-like magnetic dynamo at the AGB phase has been recently discussed by Blackman et al. (2001a), and they conclude that dynamo amplification is likely to operate in rotating AGB stars. A logical extension of this result is that a solar-like activity, including dynamo and cycles, is also expected in some AGB stars. García-Segura et al. (2001) presented 2(1/2)D magnetohydrodynamic numerical simulations of the effects of a solar-like magnetic cycle, with periodic polarity inversions, in the slow wind of an AGB star. The stellar wind was modeled with a steady mass-loss at constant velocity. This simple version of a solar-like cycle, without mass-loss variations, was able to reproduce many properties of the observed concentric rings. The shells were formed by pressure oscillations, which drive compressions in the magnetized wind. These pressure oscillations were due to periodic variations in the field intensity. The periodicity of the shells, then, was simply a half of the magnetic cycle since each shell was formed when the magnetic pressure went to zero during the polarity 
inversion. Their results showed the importance of MHD effects in the formation of the HST rings and successfully reproduced their main features. This indicates that modulated mass-loss episodes are not really necessary to generate the rings.

Acknowledgments. I thank my colleges and friends J. Franco, J. A. López, N. Langer, M. Różyczka, A. Manchado, Y.-H. Chu, Y. Terzian, N. Soker, M. Peimbert and S. Torres-Peimbert for these six, amazing years of motivating work on $\mathrm{PNe}$.

\section{References}

Aller, L. H. 1958, AJ, 63, 47

Balick, B., Wilson, J. \& Hajian, A. R. 2000, AJ, 121, 354

Begelman, M. C., \& Li, Z.-Y. 1992, ApJ, 397, 187

Bjorkman, J. E., \& Cassinelli, J. P. 1993, ApJ, 409, 429

Blackman, E. G., Frank, A., Markiel, J. A., Thomas, J. H. \& Van Horn, H. M. 2001a, Nature, 409, 485

Blackman, E. G., Frank, A., \& Welch, C. 2001b, ApJ, 546, 288

Bryce, M., López, J. A., Holloway, A. J., \& Meaburn, J. 1997, ApJ, 487, L161

Bujarrabal, V. \& Alcolea J., 1991, A\&A, 251, 536

Castor, J. I., Abbott, D. C., \& Klein, R. I. 1975, ApJ, 195, 157

Chandrasekar, S. 1961, "Hydrodynamic and hydromagnetic stability", London: Oxford at the Clarendon Press, p. 481

Chevalier, R. A., \& Luo, D. 1994, ApJ, 421, 225

Chu, Y.-H., Jacoby, G. H. \& Arendt, R. 1987, ApJS, 64, 529

Contopoulos, J. 1995, ApJ, 450, 616

Dwarkadas et al. 2002, this volume

Frank, A., Balick, B., \& Livio, M. 1996, ApJ, 471, L53

Gaensler, B. M. 1998, ApJ, 493, 781

García-Segura, G. 1997, ApJL, 489, L189

García-Segura, G., Langer, N., Różyczka, M., \& Franco, J. 1999, ApJ, 517

García-Segura, G., López, J. A. 2000, ApJ, 544, 336

García-Segura, G., López, J. A., and Franco, J. 2001, ApJ, 560, 928

García-Segura, G., Franco, J., López, J. A., Langer, N., \& Różyczka, M. 2000, Asymmetrical Planetary Nebulae II: From Origins to Microstructures, eds. Joel H. Kastner, Noam Soker, Saul A. Rappaport, A.S.P. Conference Series, 199, 235

García-Segura, G., Franco, J., López, J. A., Langer, N., \& Różyczka, M. 2002, RMxAC, in press

Gardiner, T. A., \& Frank, A. 2001, ApJ, 557, 250

Guerrero, M.A., Vázquez, R., \& López, J.A. 1998, ApJ, 117, 967

Gurzadian, G. A. 1962, In Vistas in Astronomy, vol. 5, ed. A. Beer, Pergamon, New York, p. 40

Heiligman, G. M. 1980, M.N.R.A.S., 191,761

Hrivnak, B. J., Kwok, S. \& Su, K. Y. L. 2001, AJ, 121, 2775

Iben, I. Jr. 1993, in IAU Symp. No 155 "Planetary Nebulae", ed. R. Weinberger and A. Acker, (Kluwer Academic Publishers, Dordrecht), 587

Ignace, R., Cassinelli, C. P., \& Bjorkman, J. E. 1996, ApJ, 459, 671

Ignace, R., Cassinelli, C. P., \& Bjorkman, J. E. 1998, ApJ, 505, 910 
Jackson, J. D. 1962, "Electrodynamics", eds. John Wiley \& Sons, Inc., New York, p. 482

Kahane, C. \& Jura M., 1994, A\&A, 290, 183

Kennel, C. F., \& Coroniti, F. V. 1984, ApJ, 283, 694

Kwok, S., Purton, C. R. \& Fitzgerald, P. M. 1978, ApJ 219, L125

Kwok, S., Su, K. Y. L., Hrivnak, B. J. 1998, ApJ, 501, L117

Livio, M. 1997, SSRv, 82, 389

Livio, M., \& Pringle, J. E. 1996, ApJL, 465, 55

Livio, M., Salzman, J., \& Shaviv, G. 1979, MNRAS, 188, 1

Livio, M., \& Soker, N. 1988, ApJ, 329, 746

López, J. A. 1997, in IAU Symp. No 180 "Planetary Nebulae", eds. Habing \& Lamers, (Kluwer Academic Publishers, Dordrecht), 197

Manchado, A., Guerrero, M., Stanghellini, L., \& Serra-Ricart, M. 1996a, "The IAC Morphological Catalog of Northern Galactic Planetary Nebulae", ed. Instituto de Astrofísica de Canarias

Manchado, A., Stanghellini, L., \& Guerrero, M. 1996b, ApJL, 466, L95

Manchado, A., Villaver, E., Stanghellini, L., \& Guerrero, M. A. 2000, Asymmetrical Planetary Nebulae II: From Origins to Microstructures, eds. Joel H. Kastner, Noam Soker, Saul A. Rappaport, A.S.P. Conference Series, 199, 17

Mastrodemos, N., \& Morris, M. 1998, ApJ, 497, 303

Matt, S., Balick, B., Winglee, R., \& Goodson, A. 2000, ApJ, 545, 965

Morris, M. 1987, PASP, 99, 1115

Pascoli, G. 1992, PASP, 104, 350

Pottasch, S. R. 1984, "Planetary Nebulae. A Study of Late Stages of Stellar Evolution", (D. Reidel Publishing Company, Dordrecht)

Rees, M. J., \& Gunn, J. E. 1974, M.N.R.A.S., 167, 1

Reyes-Ruiz, M., \& López, J. A. 1998, ApJ, 524, 952

Riera, A., García-Lario, P., Manchado, A., Pottasch, S. R., \& Raga, A. C. 1995, A\&A, 302,137

Różyczka, M. \& Franco, J. 1996, ApJL, 469, L127

Simis, Y. J. W., Icke, V., \& Dominik, C. 2001, A\&A, 371, 205

Schwarz, H. E., Corradi, R. L. M., \& Melnick, J. 1992, A\&A Suppl, 96, 23

Soker, N. 2000, ApJ, 540, 436

Soker, N., \& Dgani, R. 1997, ApJ, 484, 277

Terzian, Y., \& Hajian, A. R. 2000, Asymmetrical Planetary Nebulae II: From Origins to Microstructures, ed. J. H. Kastner, N. Soker, S. A. Rappaport, A.S.P. Conf. Series, 199, 33 